\title{
Local Object Patterns for the Representation and Classification of Colon Tissue Images
}

\author{
Gulden Olgun, Cenk Sokmensuer, and Cigdem Gunduz-Demir, Member, IEEE
}

\begin{abstract}
This paper presents a new approach for the effective representation and classification of images of histopathological colon tissues stained with hematoxylin and eosin. In this approach, we propose to decompose a tissue image into its histological components and introduce a set of new texture descriptors, which we call local object patterns, on these components to model their composition within a tissue. We define these descriptors using the idea of local binary patterns, which quantify a pixel by constructing a binary string based on relative intensities of its neighbors. However, as opposed to pixel-level local binary patterns, we define our local object pattern descriptors at the component level to quantify a component. To this end, we specify neighborhoods with different locality ranges and encode spatial arrangements of the components within the specified local neighborhoods by generating strings. We then extract our texture descriptors from these strings to characterize histological components and construct the bag-of-words representation of an image from the characterized components. Working on microscopic images of colon tissues, our experiments reveal that the use of these component-level texture descriptors results in higher classification accuracies than the previous textural approaches.
\end{abstract}

Index Terms-Classification, colon cancer, digital pathology, local patterns, texture, tissue image representation.

\section{INTRODUCTION}

$\mathbf{H}$ ISTOPATHOLOGICAL examination of a tissue is the routine practice to identify numerous neoplastic diseases including cancer. In this practice, pathologists examine the tissue under a microscope to find histological manifestations of a disease and then provide diagnostic information based on the findings and their interpretations. However, this practice is subject to substantial amount of subjectivity because locating and correctly interpreting the findings highly depend on expertise and experience of the pathologists.

Digital pathology emerges as a need to help the pathologists lessen the subjectivity level of their decisions. To this end,

Manuscript received February 5, 2013; revised June 17, 2013 and August 19, 2013; accepted September 5, 2013. Date of publication September 10, 2013; date of current version June 30, 2014. This work was supported by the Scientific and Technological Research Council of Turkey under the Project TÜBITAK 110E232.

G. Olgun and C. Gunduz-Demir are with the Department of Computer Engineering, Bilkent University, Ankara TR-06800, Turkey (e-mail: gulden@cs. bilkent.edu.tr; gunduz@cs.bilkent.edu.tr).

C. Sokmensuer is with the Department of Pathology, Hacettepe University Medical School, Ankara TR-06100, Turkey (e-mail: csokmens@ hacettepe edu.tr).

Color versions of one or more of the figures in this paper are available online at http://ieeexplore.iee.org.

Digital Object Identifier 10.1109/JBHI.2013.2281335 many studies have been working on developing automated diagnostic systems. These systems rely on representing a tissue image with quantitative features and using this representation for classifying the tissue. In the literature, there exist several studies that use texture descriptors to define these features. The most commonly used descriptors are those that are defined on intensity/color histograms, which quantify the first-order statistics of image pixels [1]-[3], and cooccurrence matrices, which quantify the second-order statistics among pixels [4], [5]. In addition to these, many studies make use of wavelets to define their features. Examples include the descriptors defined on multiwavelet coefficients [6] and Gabor filter responses [7]. Fractal analysis is another method used for defining texture descriptors. In this analysis, fractal dimensions are frequently used as features [8], [9]. More recent studies use local binary patterns to define additional texture descriptors [10]-[12]. They are used to quantify a pixel according to spatial arrangement of its neighbors' intensities with respect to its intensity. All these texture descriptors yield promising results. However, they are defined on pixels, directly using pixels' intensity/color values. Thus, they are susceptible to pixel-level noise and variations that are typically observed in histopathological images.

In this study, we propose a new algorithm for the effective representation and classification of images of histopathological colon tissues stained with hematoxylin and eosin. In the proposed algorithm, our main contributions are the introduction of a set of new texture descriptors, which we call local object patterns, to model composition of histological components in a tissue image and the use of this descriptor set to define the visual words of the bag-of-words representation of the image. In this algorithm, we decompose the image into component objects of multiple types and define texture of these objects using the idea of local binary patterns [13]. However, as opposed to local binary patterns defined at the pixel level, we define local object patterns on the objects at the component level. Particularly, local binary patterns are defined to quantify a pixel by constructing a binary string from the spatial arrangement of its neighbors' relative intensities. On the other hand, we define our local object patterns to quantify an object by specifying a set of neighborhoods with different locality ranges and constructing a string based on how the object's neighbors arrange in an order in each of these local neighborhoods. The motivation behind defining the local object patterns is the following: A normal tissue has the characteristic composition of histological components and cancer causes deviations from it. Therefore, components (objects) belonging to similar regions in normal and cancerous tissues are expected to have neighbors of different types in specified neighborhoods as a part of their composition. Thus, the difference 
in neighbor distributions can be used to differentiate such components, the distribution of which can be used to differentiate normal and cancerous tissues. Our experiments on 3236 microscopic images of colon tissues demonstrate that our proposed texture descriptors are effective to obtain better classification accuracies compared to previous texture definitions.

The proposed algorithm mainly differs from the previous texture-based tissue classification studies in the following two aspects: First, it defines its texture descriptors on higher level component objects instead of defining them at the pixel level. Second, our algorithm uses the component-level texture descriptors to quantize the objects and constructs the bag-of-words representation of an image from its quantized objects, instead of directly using texture descriptors for representing the image. Our experiments show that these differences make our algorithm less susceptible to pixel-level noise and variations observed in the overall image.

In the literature, there exist structural approaches that also use histological components to represent a tissue image. These approaches commonly construct a graph on these components and use graph descriptors for image classification. Earlier studies construct their graphs on only nucleus tissue components using different techniques such as Delaunay triangulations [7], [14], minimum spanning trees [15], and probabilistic graph generations [16]. In our more recent study [17], we construct a graph on tissue components of different types and color graph edges based on the types of their end nodes. Different than our proposed texture descriptors defined within objects' local neighborhoods, these previous structural approaches usually use a global graph representation for the entire image and extract global graph descriptors for its quantification.

In our previous studies [18]-[20], we defined other objectbased descriptors. However, the definitions of these descriptors are completely different. The currently proposed local object patterns encode the object's local neighborhood information by constructing a set of strings and they are used for tissue image classification. Different than this current work, we defined object-based cooccurrence and run-length matrices in [18] and [19], respectively, and introduced a uniformity metric on objects in [20]. Moreover, our previous studies did not make any classification but focused on unsupervised tissue image segmentation.

\section{Methodology}

Our algorithm decomposes a tissue image into its histological components, characterizes them with the newly introduced local object pattern descriptors, and uses this characterization for classification of tissue images. The details of these steps are explained in the following sections.

\section{A. Tissue Image Decomposition}

We model a tissue image $I$ by approximately representing its histological components with a set of circular objects $O$

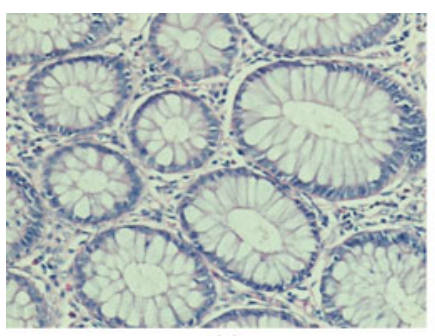

(a)

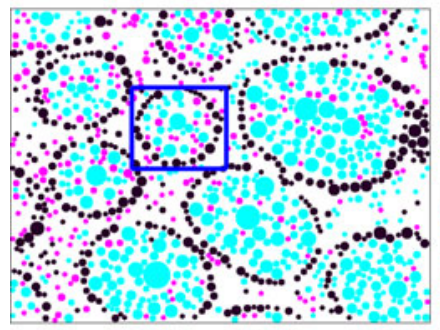

(c)

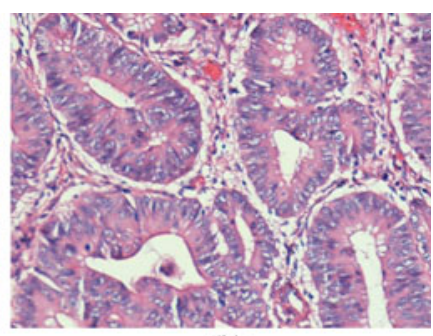

(b)

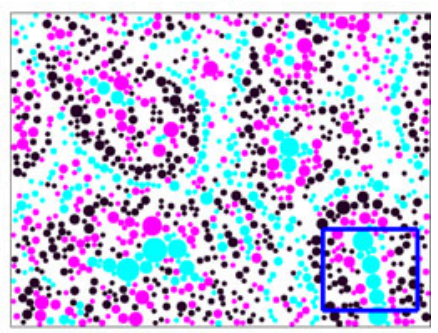

(d)
Fig. 1. Examples of (a) normal and (b) cancerous tissue images. Objects located on the (c) normal and (d) cancerous tissue images. Here, purple, pink, and white objects are shown as purple, pink, and cyan circles, respectively.

$(I)=\left\{o_{i}\right\}$. We represent each object $o_{i}$ by its coordinates $\left(x_{i}, y_{i}\right)$ and its type $t_{i} \in\{\text { purple, pink, white }\}^{1}$.

To define this object set, we first separate hematoxylin and eosin channels of the image $I$ by applying color deconvolution [21]. We then use these channels to quantize pixels into three groups (purple, pink, and white). Let $h_{p}$ and $e_{p}$ be the values of pixel $p$ in the hematoxylin and eosin channels, respectively, and $h_{\text {avg }}$ and $e_{\text {avg }}$ be the average pixel values in these channels. We label pixel $p$ as purple if $h_{p} \leq h_{\text {avg }}$, pink if $h_{p}>h_{\text {avg }}$ and $e_{p} \leq e_{\text {avg }}$, and white if $h_{p}>h_{\text {avg }}$ and $e_{p}>e_{\text {avg. }}$. We finally apply the circle-fit algorithm [20] on the pixels of each group separately to locate a set of circular objects. This algorithm iteratively locates objects starting from the largest one as long as the radii of located circles are greater than threshold $r_{\min }$. The centroid of an object determines its coordinates $\left(x_{i}, y_{i}\right)$ and the pixel group on which it is located determines its type $t_{i}$. Fig. 1 shows example tissue images and their located objects.

In our model, we use an approximate representation instead of finding exact locations of histological components because their exact localization gives rise to a quite difficult segmentation problem. Thus, there may be one-to-one or many-to-one relation between objects and components. For example, a purple object usually corresponds to a single nucleus, whereas a group of white objects that form a clique corresponds to a lumen region. The proposed local binary patterns are also effective to model such many-to-one relations.

\footnotetext{
${ }^{1}$ These types correspond to the three main colors in a hematoxylin-and-eosin stained tissue. Particularly, cell nuclei correspond to purple; stroma, stromal cells' cytoplasms, and mucin-poor epithelial cells' cytoplasms correspond to pink; and lumina and mucin-rich epithelial cells' cytoplasms correspond to white. Since there are multiple components corresponding to the same type, we hereinafter refer to them as purple, pink, and white, to keep the manuscript simpler and easier to read.
} 

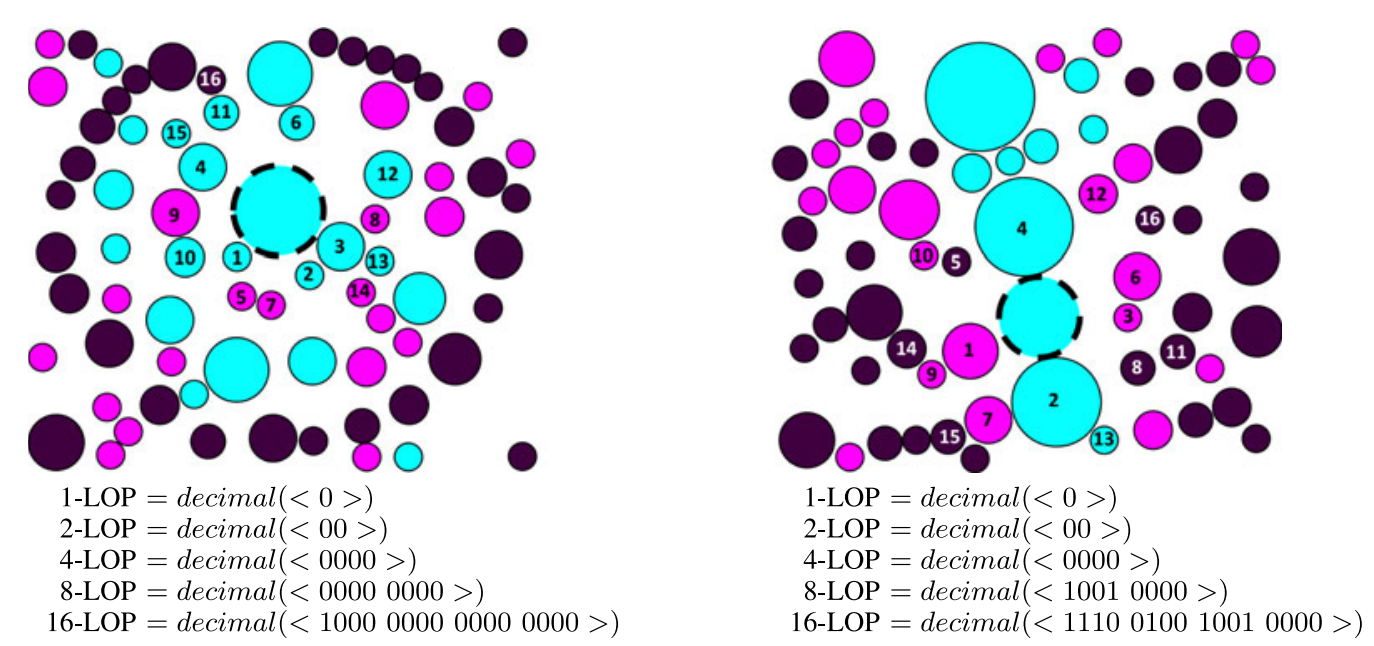

Fig. 2. Extracting local object patterns for the objects with dashed borders. Here $m$ is selected as 4, and thus $\mathcal{S}=\bigcup_{j=0}^{4} 2^{j}$-LOP. 16-nearest neighbors of the selected objects are indicated on the examples with their orders.

\section{B. Local Object Patterns}

For object $o_{i}$, we define the $n$th local object pattern $n$-LOP $\left(o_{i}\right)$ as follows: We select $n$-nearest neighbors of $o_{i}$ and order them according to distances from their coordinates to $\left(x_{i}, y_{i}\right)$. Let $N\left(o_{i}\right)=<o_{i n}, \ldots, o_{i j}, \ldots, o_{i 1}>$ be the ordered neighbor set of $o_{i}$, where $o_{i n}$ and $o_{i 1}$ are its farthest and closest neighbors, respectively. We form a binary string $B\left(o_{i}\right)=<$ $b_{i n}, \ldots, b_{i j}, \ldots, b_{i 1}>$ considering types $t_{i j}$ of the selected neighbors. In this string

$$
b_{i j}= \begin{cases}1 & \text { if } t_{i j} \in\{\text { purple }\} \\ 0 & \text { if } t_{i j} \in\{\text { pink, white }\} .\end{cases}
$$

Then, we define $n$-LOP $\left(o_{i}\right)$ as the decimal equivalent of binary string $B\left(o_{i}\right)$. Note that this descriptor provides rotation invariance since objects are ordered based on their distances to object $o_{i}$ and its value does not change with arbitrary rotations of the image.

We define local object patterns for an object to quantify the spatial arrangement of its neighbors' types found in a local neighborhood. In our model, we extract a set of $m+1$ patterns using different neighborhoods. Particularly, this set includes $\mathcal{S}=\bigcup_{j=0}^{m} 2^{j}$-LOP. We will use this pattern set to label each object with a new type and use the new types' frequency in an image for its classification. We will explain these steps in the next section.

\section{Bag-of-Words Representation and Classification}

In this study, we use the observation that components belonging to similar regions in normal and cancerous tissue images show different characteristics in their neighbor distributions. To differentiate these components (objects), we define new object types based on local object patterns, which quantify the neighbor distributions. For example, Fig. 2 illustrates the extraction of local object patterns for the objects with dashed borders. We select these objects such that they both belong to luminal regions; we crop these regions from normal and cancerous tissue images as shown in Fig. 1. Fig. 2 shows that although lower order patterns are the same for the two selected objects, their higher order patterns show differences, which can be used to differentiate these objects.

We define the new object types as follows: For each original type $t_{i} \in$ \{purple, pink, white $\}$, we separately cluster objects of the corresponding type into $k$ groups running the k-means algorithm on local object patterns of these objects. Thus, we learn $k$ clustering vectors $V_{\text {purple }}=\left\{v_{1}, \ldots, v_{k}\right\}$ for the purple type, $k$ clustering vectors $U_{\text {pink }}=\left\{u_{1}, \ldots, u_{k}\right\}$ for the pink type, and $k$ clustering vectors $W_{\text {white }}=\left\{w_{1}, \ldots, w_{k}\right\}$ for the white type. Then, for a given image, we relabel each object $o_{i}$ with a new type $t_{i}^{\prime}$ based on its original type $t_{i}$ and the corresponding set of the clustering vectors $V_{\text {purple }}, U_{\text {pink }}$, or $W_{\text {white }}$; that is, we take the clustering vector set $V_{\text {purple }}, U_{\text {pink }}$, or $W_{\text {white }}$ according to the original type $t_{i} \in\{$ purple, pink, white $\}$ and assign the object $o_{i}$ to the closest cluster in this set. Since components (objects) of normal and cancerous tissue images show different neighbor distributions, they are expected to be relabeled with different types $t_{i}^{\prime}$. Thus, we use the distribution of these new types to represent an image. To this end, we extract the bagof-words representation on the frequency of objects' new types and classify the image using a linear kernel support vector machine (SVM) classifier. Note that, in this study, we use the SVM implementation provided by [22]; this implementation uses the one-against-one strategy for multiclass classifications.

\section{EXPERIMENTS}

\section{A. Dataset}

We conduct our experiments on 3236 microscopic images of hematoxylin and eosin-stained colon tissues of 258 patients. Images are taken using a Nikon Coolscope Digital Microscope with a $20 \times$ objective lens and at $640 \times 480$ pixel resolution. Images are divided into training and test sets such that they contain images of different patients. Three different classes are used to label each of these images: normal, low-grade cancerous, 
and high-grade cancerous. ${ }^{2}$ The training set contains 510 normal, 859 low-grade cancerous, and 275 high-grade cancerous images of 129 patients. The test set contains 491 normal, 844 low-grade cancerous, and 257 high-grade cancerous images of the remaining 129 patients.

\section{B. Comparisons}

We compare our results with the results of previous textural and structural methods.

1) Textural Methods: We first use four texture descriptors, all defined at the pixel level, to understand the effectiveness of defining texture descriptors at the component level. These pixel-level descriptors are extracted using intensity histograms, gray-level cooccurrence matrices, Gabor filters, and local binary patterns. Similar to ours, all these algorithms use linear kernel SVM classifiers.

The IntensityHistogram [23] and CooccurrenceMatrix [4] descriptors are the first- and second-order statistics calculated on gray-level intensities of image pixels. Particularly, the IntensityHistogram descriptors include mean, standard deviation, kurtosis, and skewness of a gray-level intensity histogram. The CooccurrenceMatrix descriptors include energy, entropy, contrast, homogeneity, correlation, dissimilarity, inverse difference moment, and maximum probability of gray-level cooccurrence matrices extracted at eight orientations. In our experiments, we first calculate these descriptors on entire images. However, it is commonly difficult to find a constant texture over an entire image since the tissue image may contain subregions irrelevant to classification. Thus, we also implement the grid-based variants of these descriptors. In these grid-based variants, we divide the image into fixed sized grids, extract an histogram (or a cooccurrence matrix) on each grid, calculate descriptors on the grid histograms (or grid cooccurrence matrices), and average the grid descriptors all over the image.

To extract the GaborFilter descriptors, we first convolve an image with log-Gabor filters in six orientations and four scales [24]. Then, for each scale, we average the responses of different orientations to obtain rotation invariance, and calculate average, standard deviation, minimum-to-maximum ratio, and mode descriptors [7] on this average. Likewise, we implement the grid-based variant of these descriptors.

The LocalBinaryPattern descriptors include histogram frequencies. We compute this histogram on the outputs of a uniform local binary pattern (LBP) operator [13] applied on image pixels. For each pixel, the LBP operator outputs a binary string by comparing the pixel's gray-scale intensity with those of its eight neighbors; it outputs 1 if its intensity is lower and 0 otherwise. It then assigns the pixel to an histogram bin based on the number of consecutive 1's in this binary string. This operator is called uniform if it constructs the histogram on only the pixels whose binary strings contain at most two bitwise $0 / 1$ transitions in their circular chain. We calculate an additional

\footnotetext{
${ }^{2}$ The images were labeled by Prof. C. Sokmensuer, MD, who is specialized in colorectal carcinomas and has been practicing pathology for nearly 20 years. Each image was shown to him for at least two different times and at the end, he decided on the image label considering his multiple decisions.
}

bin for keeping frequencies of pixels with nonuniform strings. In our experiments, we extract these descriptors from the histogram constructed on all pixels. Here, we did not implement its grid-based variant because calculating histograms on pixels of equal-sized grids and averaging their histogram frequencies is equivalent to calculating an histogram on all pixels and using its frequencies.

Instead, we make use of local binary patterns to implement the pixel-based counterpart of our algorithm, which we call PixelBasedAlgorithm. This algorithm follows exactly the same steps of our algorithm except its descriptor definition step. Particularly, it decomposes a tissue image into a set of circular objects, defines descriptors on the objects, clusters the objects based on their descriptors to find their new types, and uses the new types' frequency in a linear kernel SVM classifier. Here, different from our proposed algorithm, which uses local object patterns as the descriptors, the PixelBasedAlgorithm uses local binary patterns. To this end, it locates a square window at the center of each object and calculates local binary patterns of this window to find the descriptors of the object. We use this comparison in our experiments to understand the effectiveness of defining component-level local object patterns.

Additionally, we use the resampling-based Markovian model $(R M M)$ that we implemented in our previous work [25]. The $R M M$ obtains multiple samples of an image, labels each sample using discrete Markov models, and votes the samples' labels to classify the image. To obtain an image sample, it generates a sequence on the randomly selected points, which are characterized by texture descriptors and ordered based on proximity. These descriptors include the histogram of quantized pixels and the $J$-value texture measure [26].

All these textural methods except the PixelBasedAlgorithm extract their features directly on image pixels. Thus, their complexity is polynomially bounded by the number $N_{p}$ of the pixels in an image. On the other hand, the PixelBasedAlgorithm first locates objects, complexity of which is also polynomial with respect to $N_{p}$, and then extracts its features for each object considering its neighboring pixels. Likewise, our proposed algorithm locates the objects and extracts its features for each object but this time considering the neighboring objects. Since the object number is much lower than the pixel number, the feature extraction of both of these algorithms is also polynomially bounded by $N_{p}$.

2) Structural Methods: We first compare our algorithm with two structural methods that use global graph features: DelaunayTriangulation and ColorGraph. The former constructs a Delaunay triangulation on purple circular objects and extracts features including average degree, average clustering coefficient, and diameter, as well as average, standard deviation, minimumto-maximum ratio, and disorder of edge lengths and triangle areas [7]. The latter also constructs a Delaunay triangulation, but this time on all types of circular objects, and colors triangle edges based on the types of their end nodes. This method extracts colored versions of average degree, average clustering coefficient, and diameter features [17]. These two methods use linear kernel SVM classifiers. 
The GraphWalk method is another sampling approach [27]. It represents an image generating a set of subgraphs, classifies each subgraph based on its edge distribution, and votes the subgraphs' classes to classify the entire image. This method obtains the subgraphs by first constructing a graph on the attributed components in the entire image and then sampling this graph with the breadth first search algorithm. It also uses a linear kernel SVM to classify the subgraphs.

The last method is the HybridModel that we recently developed in our research group [28]. This model first represents an image with an attributed graph and defines smaller query graphs as a reference to normal gland structures. It then selects the regions of the image whose subgraphs are most structurally similar to the query graphs based on graph edit distances. Using the graph edit distances of the selected regions as well as their texture descriptors, it classifies the image by a linear kernel SVM.

These structural methods decompose tissue images into objects and define their features on the object representation. Thus, after image decomposition, the complexity is polynomial with respect to the number of the objects. However, since the objects are located on image pixels, the overall time complexity of the feature extraction is polynomially bounded by the number $N_{p}$ of the pixels.

\section{Parameter Selection}

The proposed algorithm has three model parameters: minimum circle radius $r_{\min }$, highest degree $m$ (highest order $2^{m}$ ) of local object patterns, and cluster number $k$. Additionally, there is parameter $C$ for the linear kernel SVM. In our experiments, we consider all possible combinations of $r_{\min } \in\{3,4,5\}, m \in\{2,3,4,5\}, k \in\{5,10,20,30\}$, and $C \in\{1,2, \ldots, 9,10,20, \ldots, 90,100,150, \ldots, 950,1000\}$ as candidate sets and select the one that gives the highest accuracy when we use threefold cross validation on the training set. The selected parameters are $r_{\text {min }}=4, m=4, k=20$, and $C=90$. For the comparison methods, we also use threefold cross validation to select their parameters.

\section{Results}

We report the test set results obtained by our proposed LocalObjectPattern algorithm and the comparison methods in Table I. This table shows that the proposed algorithm gives high $(>90 \%)$ accuracies for all classes, leading to the highest overall accuracy. We also provide the confusion matrix for the proposed LocalObjectPattern algorithm in Table II. This table depicts that most of the confusions occur in between low-grade and high-grade cancerous tissues. This is indeed consistent with the current practice, in which incorrect decisions are typically observed in grading especially when tissues lie at the boundary between low-grade and high-grade cancer. This table also shows that confusions rarely occur in between normal- and high-grade cancerous tissues.

The PixelBasedAlgorithm takes the same steps of our algorithm except the definition of its descriptors. It uses local binary pattern descriptors, which are the pixel-based counterpart of
TABLE I

Test Set Results of the Proposed LocalObjectPattern Algorithm AND THE COMPARISON METHODS

\begin{tabular}{|l|c|c|c|c|}
\hline & Normal & Low & High & Overall \\
\hline LocalObjectPattern & 95.32 & 92.54 & 90.27 & 93.03 \\
\hline Textural methods & & & & \\
\hline IntensityHistogram & 80.65 & 69.55 & 70.04 & 73.05 \\
\hline IntensityHistogramGrid & 78.82 & 74.17 & 78.60 & 76.32 \\
\hline CooccurrenceMatrix & 83.10 & 81.64 & 77.82 & 81.47 \\
\hline CooccurrenceMatrixGrid & 87.58 & 84.12 & 85.60 & 85.43 \\
\hline GaborFilter & 88.59 & 80.09 & 77.43 & 82.29 \\
\hline GaborFilterGrid & 91.24 & 82.23 & 78.60 & 84.42 \\
\hline LocalBinaryPattern & 92.67 & 73.46 & 80.54 & 80.53 \\
\hline PixelBasedAlgorithm & 94.50 & 90.17 & 76.65 & 89.32 \\
\hline RMM & 95.64 & 87.77 & 88.56 & 90.32 \\
\hline Structural methods & & & & \\
\hline DelaunayTriangulation & 89.61 & 71.56 & 87.55 & 79.71 \\
\hline ColorGraph & 92.67 & 82.46 & 86.38 & 86.24 \\
\hline GraphWalk & 93.48 & 90.40 & 92.61 & 91.71 \\
\hline HybridModel & 96.95 & 88.27 & 96.11 & 92.21 \\
\hline
\end{tabular}

TABLE II

CONFusion Matrix of the Proposed LocalObJectPattern ALGORITHM FOR THE TEST SET

\begin{tabular}{|c|c|c|c|c|}
\hline & \multicolumn{3}{|c|}{ Computed } \\
\hline & & Normal & Low & High \\
\hline \multirow{3}{*}{ 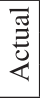 } & Normal & 468 & 19 & 4 \\
\hline & Low & 16 & 781 & 47 \\
\hline & High & 0 & 25 & 232 \\
\hline
\end{tabular}

our proposed local object patterns. Comparison of these two algorithms reveals that this new definition of local patterns on objects (tissue components) provides more effective representation, resulting in better accuracies especially for classifying high-grade cancerous tissue images.

The IntensityHistogram, CooccurrenceMatrix, GaborFilter, and LocalBinaryPattern algorithms extract global texture descriptors on an entire image (using all image pixels) whereas the $R M M$ uses pixel-based texture descriptors locally defined for the selected points. On the other hand, the proposed algorithm extracts texture descriptors for each object in a local neighborhood defined by the distance from this object to its $2^{m}$-nearest neighbor. The results show that using object-based textures is more effective to obtain higher accuracies. The gridbased variants improve results; however, they are still lower than the results of the proposed algorithm.

The DelaunayTriangulation and ColorGraph methods also employ circular objects obtained by tissue image decomposition but use a structural representation. Table I shows that they result in lower accuracies than the proposed algorithm. Both of these methods use global properties of the graph defined for an entire image in their classification. On the other hand, the GraphWalk and HybridModel methods use characteristics of local graphs defined for the selected regions of the image. The use of locality improves the overall classification accuracy. However, the proposed LocalObjectPattern algorithm still yields better results. As an interesting future work, it is possible to use the proposed local object patterns as the texture descriptors of the HybridModel. This might further increase the classification accuracy. 


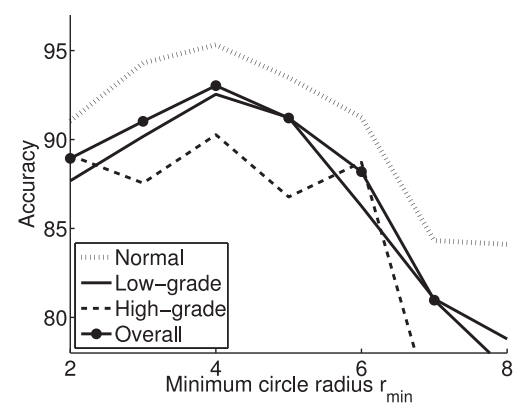

(a)

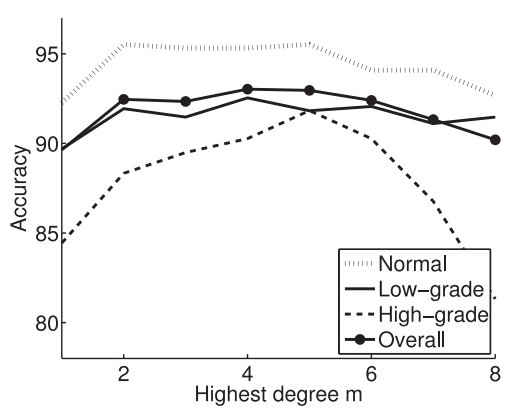

(b)

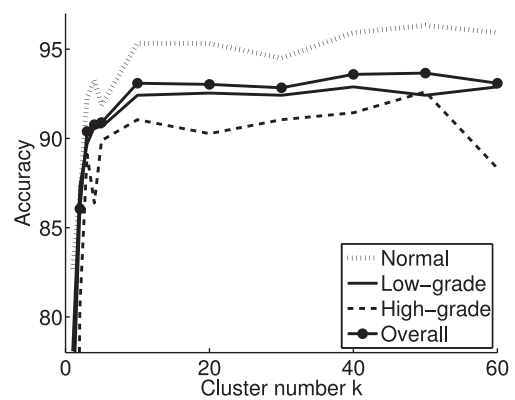

(c)

Fig. 3. Test set accuracies as a function of the model parameters: (a) minimum circle radius $r_{\min }$, (b) highest degree $m$ of local object patterns, and (c) cluster number $k$.

In our experiments, we also investigate the effects of dividing images into training and test sets. To this end, we also apply tenfold cross validation for our algorithm and obtain the average results over the test sets of the ten folds. For our algorithm, the percentages are $96.00 \pm 1.88$ for the normal class, $90.84 \pm 1.28$ for the low-grade cancerous class, $94.55 \pm 1.88$ for the highgrade cancerous class, and $93.05 \pm 0.85$ for the overall accuracy. These results indicate that the overall accuracy obtained using tenfold cross validation is quite similar to the overall accuracy obtained on the test set. Moreover, the standard deviation of the overall accuracy is low, indicating that this accuracy is not so much dependent on the division of image samples into training and test sets.

\section{E. Parameter Analyis}

We investigate the effects of each parameter on the performance of our proposed LocalObjectPattern algorithm. To this end, for each parameter, we fix the other two and analyze accuracy as a function of this parameter. Fig. 3 presents these analyses.

The first parameter is the minimum circle radius $r_{\min }$, which is used as a threshold on objects' radii in tissue image decomposition. Larger values of this parameter result in not defining smaller objects, which may correspond to important histological components. For example, in a typical image, nuclei's radii are relatively small compared to other components. Using larger thresholds may result in not defining purple objects corresponding to these nuclei. This lowers accuracy, as seen in Fig. 3(a). On the other hand, smaller threshold values may lead to noisy false objects, which, in turn, leads to defining false neighbors in extracting local object pattern descriptors. This also decreases classification accuracy.

In the proposed algorithm, we use a set of local object patterns $\mathcal{S}=\bigcup_{j=0}^{m} 2^{j}$-LOP to characterize an object. The second parameter $m$ is the highest degree, which determines the size of this set (and also the local object pattern with the highest order). As a consequence, it determines the size of neighborhood from which descriptors are extracted. When larger values are used, this neighborhood spans larger regions. This causes to lose locality in descriptor definition. On the other hand, when smaller values are used, characterization of an object mainly relies on composition of its closer neighbors. This may result in defining nondistinctive descriptors for objects belonging to images of different classes. Both of these conditions lower classification accuracies, as observed in Fig. 3(b).

After extracting the descriptors, we separately quantize objects of each type (each of the purple, pink, and white types) into clusters. The last parameter is the number $k$ of these clusters. Smaller cluster numbers may not allow defining distinctive new object types, which decreases accuracy. Increasing this parameter increases the number of words in the bag-of-words representation; this increases the number of features used in classification. Our analyses reveal that this increase only slightly affects accuracy [see Fig. 3(c)]; we attribute this small change to the curse-of-dimensionality phenomenon in classification.

\section{CONCLUSION}

This study presents a new algorithm for representing and classifying colon tissue images. In this algorithm, we introduce a set of new high-level texture descriptors called local object patterns. We define these descriptors on tissue objects, which approximately represent histological tissue components. To this end, we specify a set of neighborhoods with different locality ranges and construct a binary string for each of these neighborhoods to encode spatial arrangements of the objects within the specified local neighborhoods. We then characterize tissue objects using the decimal equivalents of the binary strings as descriptors and construct bag-of-words representation of an image from its characterized objects. We test our proposed algorithm on 3236 microscopic images of colon tissues stained with hematoxylin and eosin. Our experiments demonstrate that our algorithm, which uses local object pattern descriptors, lead to higher classification accuracies than its pixel-based counterparts.

The proposed algorithm constructs a binary string to encode objects' composition in a specified local neighborhood. In this binary string, purple objects, which usually correspond to nucleus components, are represented with 1 and the others with 0 . Instead of this binary representation, one could consider constructing ternary strings where pink and white objects are represented with different values. Besides, the proposed algorithm computes the local object pattern descriptors by converting the binary strings to their decimal equivalents. It is also possible 
to obtain these descriptors directly from the strings. Exploring these possibilities could be considered as future research directions for this study.

\section{REFERENCES}

[1] A. Tabesh, M. Teverovskiy, H. Y. Pang, V. P. Kumar, D. Verbel, A. Kotsianti, and O. Saidi, "Multifeature prostate cancer diagnosis and Gleason grading of histological images," IEEE Trans. Med. Imag., vol. 26, no. 10, pp. 1366-1378, Oct. 2007.

[2] R. Rahmadwati, G. Naghdy, M. Ros, and C.Todd, "Computer aided decision support system for cervical cancer classification," in Proc. SPIE, Applications of Digital Image Processing XXXV, Oct. 2012, vol. 8499, p. 849919.

[3] F. Bunyak, A. Hafiane, and K. Palaniappan, "Histopathology tissue segmentation by combining fuzzy clustering with multiphase vector level sets," Adv. Exp. Med. Biol., vol. 696, pp. 413-424, 2011.

[4] A. N. Esgiar, R. N. G. Naguib, B. S Sharif, M. K. Bennett, and A. Murray, "Microscopic image analysis for quantitative measurement and feature identification of normal and cancerous colonic mucosa," IEEE Trans. Inf. Technol. Biomed., vol. 2, no. 3, pp. 197-203, Sep. 1998.

[5] S. Doyle, M. Feldman, J. Tomaszewski, and A. Madabhushi, "A boosted Bayesian multi-resolution classifier for prostate cancer detection from digitized needle biopsies," IEEE Trans. Biomed. Eng., vol. 59, no. 5, pp. 1205-1218, May 2012.

[6] K. Jafari-Khouzani and H. Soltanian-Zadeh, "Multiwavelet grading of pathological images of prostate," IEEE Trans. Biomed. Eng., vol. 50, no. 6, pp. 697-704, Jun. 2003.

[7] S. Doyle, S. Agner, A. Madabhushi, M. Feldman, and J. Tomaszewski, "Automated grading of breast cancer histopathology using spectral clustering with textural and architectural image features," in Proc. Biomed Imag.: Nano Macro, 2008, pp. 496-499.

[8] A. N. Esgiar, R. N. G. Naguib, B. S. Sharif, M. K. Bennett, and A. Murray, "Fractal analysis in the detection of colonic cancer images," IEEE Trans. Inf. Technol. Biomed., vol. 6, no. 1, pp. 54-58, Mar. 2002.

[9] P.-W. Huang and C.-H. Lee, "Automatic classification for pathological prostate images based on fractal analysis," IEEE Trans. Med. Imag., vol. 28, no. 7, pp. 1037-1050, Jul. 2009.

[10] H. Qureshi, O. Sertel, N. Rajpoot, R. Wilson, and M. N. Gurcan, "Adaptive discriminant wavelet packet transform and local binary patterns for meningioma subtype classification," in Proc. Med. Image Comput. Comput.Assisted Intervent., 2008, pp. 196-204.

[11] O. Sertel, J. Kong, H. Shimada, U. V. Catalyurek, J. H. Saltz, and M.N. Gurcan, "Computer-aided prognosis of neuroblastoma on whole slide images: Classification of stromal development," Pattern Recogn., vol. 42, no. 6, pp. 1093-1103, 2009.

[12] Y. Zhang, B. Zhang, F. Coenen, and W. Lu, "Breast cancer diagnosis from biopsy images with highly reliable random subspace classifier ensembles," Mach. Vis. Appl., pp. 1-16, 2012.

[13] T. Ojala, M. Pietikainen, and T. Maenpaa, "Multiresolution gray-scale and rotation invariant texture classification with local binary patterns," IEEE Trans. Pattern Anal. Mach. Intell., vol. 24, no. 7, pp. 971-987, Jul. 2002.

[14] A. N. Basavanhally, S. Ganesan, S. Agner, J. P. Monaco, M. D. Feldman, J. E. Tomaszewski, G. Bhanot, and A. Madabhushi, "Computerized imagebased detection and grading of lymphocytic infiltration in HER2+ breast cancer histopathology," IEEE Trans. Biomed. Eng., vol. 57, no. 3, pp. 642653, Mar. 2010
[15] H.-K. Choi, T. Jarkrans, E. Bengtsson, J. Vasko, K. Wester, P.-U. Malmstrom, and C. Busch, "Image analysis based grading of bladder carcinoma. Comparison of object, texture, and graph based methods and their reproducibility," Anal. Cell. Pathol., vol. 15, pp. 1-18, 1997.

[16] C. Demir, S. H. Gultekin, and B. Yener, "Learning the topological properties of brain tumors," IEEE ACM Trans. Comput. Biol., vol. 2, no. 3, pp. 262-270, Jul./Sep. 2005.

[17] D. Altunbay, C. Cigir, C. Sokmensuer, and C. Gunduz-Demir, "Color graphs for automated cancer diagnosis and grading," IEEE Trans. Biomed. Eng., vol. 57, no. 3, pp. 665-674, Mar. 2010.

[18] A. B. Tosun and C. Gunduz-Demir, "Graph run-length matrices for histopathological image segmentation," IEEE Trans. Med. Imag., vol. 30, no. 3, pp. 721-732, Mar. 2011

[19] A. C. Simsek, A. B. Tosun, C. Aykanat, C. Sokmensuer, and C. GunduzDemir, "Multilevel segmentation of histopathological images using cooccurrence of tissue objects," IEEE Trans. Biomed. Eng., vol. 59, no. 6, pp. 1681-1690, Jun. 2012.

[20] A. B. Tosun, M. Kandemir, C. Sokmensuer, and C. Gunduz-Demir, "Object-oriented texture analysis for the unsupervised segmentation of biopsy images for cancer detection," Pattern Recognit., vol. 42, no. 6, pp. 1104-1112, 2009.

[21] A. C. Ruifrok and D. A. Johnston. (2001). Quantification of histochemical staining by color deconvolution. Anal. Quant. Cytol. Histol. [Online]. 23, pp. 291-299. Available: Source code is available at http:// www.dentistry.bham.ac.uk/landinig/software/cdeconv/cdeconv.html

[22] C.-C. Chang and C.-J. Lin. (2011). LIBSVM: A library for support vector machines. ACM Trans. Intell. Syst. Tech. [Online]. 2(3), pp. 1-27. Available: http://www.csie.ntu.edu.tw/ cjlin/libsvm

[23] M. Wiltgen, A. Gerger, and J. Smolle, "Tissue counter analysis of benign common nevi and malignant melanoma," Int. J. Med. Inform., vol. 69, pp. 17-28, 2003.

[24] P. Kovesi, Code for convolving an image with a bank of logGabor filters, [Online]. Available: http://www.csse.uwa.edu.au/ ppk/ Research/MatlabFns/PhaseCongruency/gaborconvolve.m

[25] E. Ozdemir, C. Sokmensuer, and C. Gunduz-Demir, "A resampling-based Markovian model for automated colon cancer diagnosis," IEEE Trans. Biomed. Eng., vol. 59, no. 1, pp. 281-289, Jun. 2012.

[26] Y. Deng and B. S. Manjunath, "Unsupervised segmentation of colortexture regions in images and video," IEEE Trans. Pattern Anal. Mach. Intell., vol. 23, no. 8, pp. 800-810, Aug. 2001.

[27] G. Olgun, C. Sokmensuer, and C. Gunduz-Demir, "Graph walks for classification of histopathological images," in Proc. Biomed. Imag.: Nano Macro, 2013.

[28] E. Ozdemir and C. Gunduz-Demir, "A hybrid classification model for digital pathology using structural and statistical pattern recognition," IEEE Trans. Med. Imag., vol. 32, no. 2, pp. 474-483, Feb. 2013.

Authors' photographs and biographies not available at the time of publication. 